\title{
APPROXIMATION ON WILD JORDAN CURVES
}

\author{
JACOB KOREVAAR AND HERBERT ALEXANDER $\dagger$
}

\section{Introduction}

Throughout this paper, the letter $\gamma$ denotes a (positively oriented) Jordan curve in the $z$-plane which contains the origin in its interior. By a well-known result of Walsh $[7,8]$, all continuous functions on such a curve $\gamma$ are uniform limits of polynomials in $z$ and $1 / z$. In other words, the integral powers $z^{n}, n=0, \pm 1, \ldots$ form a spanning set for the Banach space $C(\gamma)$.

One may ask if all powers $z^{n}$ are required for such a spanning set. If $\gamma$ has finite length $L$, the answer is yes: by Cauchy's theorem,

$$
|2 \pi i|=\left|\int_{\gamma}\left(z^{-1}-\sum_{n \neq-1} c_{n} z^{n}\right) d z\right| \leqslant\left\|z^{-1}-\sum_{n \neq-1} c_{n} z^{n}\right\| \cdot L
$$

for every finite sum $\sum_{n \neq-1} c_{n} z^{n}$, and hence the power $z^{-1}$ has positive distance (actually equal to $2 \pi / L, c f$. [2]) to the closed span of the other powers $z^{\prime \prime}$. The same is true for every integral power $z^{s}$.

In 1957, Wermer [9] observed that for every Jordan curve $\gamma$ of infinite length, (at least) one power of $z$ is superfluous (the powers $z^{n}, n \neq s$, form a spanning set for $C(\gamma)$ ). A different proof and an extension of this result have been given by Pia Pfluger and the first author [2]. They constructed curves for which precisely $p(\geqslant 1)$ powers are superfluous, as well as a curve for which any finite set of powers can be omitted. In the present note, we obtain simple Jordan curves $\gamma^{*}$ with the following property. For every (increasing) sequence of positive integers $\left\{p_{k}\right\}$ of positive density, the set of powers

$$
\left\{z^{n}, n=\ldots,-p_{2},-p_{1}, 0,1,2, \ldots\right\}
$$

spans the space $C\left(\gamma^{*}\right)$. We observe further that for any such curve, $C\left(\gamma^{*}\right)$ must even have spanning sets (1.1) corresponding to certain sequences $\left\{p_{k}\right\}$ of density zero.

One is thus led to ask: Could there be a curve $\gamma$ such that (1.1) is a spanning set for $C(\gamma)$, no matter how the (infinite) sequence $\left\{p_{k}\right\}$ is selected?

\section{Measures orthogonal to (1.1) as boundary values of holomorphic differentials}

Let $D$ be the interior of our curve $\gamma$ in the $z$-plane, $\Delta$ the open unit disc in the $w$-plane. We let $w=\Phi(z)$ be the 1-1 conformal map of $D$ onto $\Delta$, normalized so that $\Phi(0)=0, \Phi^{\prime}(0)>0$. By the theorem of Carathéodory and Osgood, this map has a continuous $1-1$ extension to $\bar{D}$ (onto $\bar{\Delta}$ ), which we also call $\Phi$. The inverse map will be called $z=\Psi(w)$. For $0<r \leqslant 1$, we denote the positively oriented circle $|w|=r$ by $\Gamma_{r}$, its image $\Psi\left(\Gamma_{r}\right)$ in the $z$-plane by $\gamma_{r}$ (so that $\gamma_{1}=\gamma$ ).

Received 16 June, 1975.

† Work supported, in part, by NSF grants GP38584 and GP38214.

[J. LONDON MATH. SOC. (2), 13 (1976), 317-322] 
LemMa. Let $\mu$ be a complex Borel measure on $\gamma$ orthogonal to the powers $z^{\prime \prime}, n \geqslant 0$ and $z^{-p_{k}}, k=1,2, \ldots$, where the $p_{k}$ 's are distinct positive integers. Then there is a (unique) holomorphic function $g$ on $D$ with the following properties:

(i) $\mu$ is a weak boundary value of $d g$ : for every Borel set $A \subseteq \gamma$, one has

$$
\mu(A)\left(=\int_{A} d \mu(z)\right)=\lim _{r \uparrow 1} \int_{A_{r}} d g(z),
$$

where $A_{r}$ is the set obtained by "pulling $A$ back to $\gamma_{r}$ ": $A_{r}=\Psi\{r \Phi(A)\}$.

(ii) Near $z=0, g(z)$ has the form

$$
\sum b_{j} z^{q j}
$$

where $\left\{q_{j}\right\}$ is the set of positive integers complementary to the $\operatorname{set}\left\{p_{k}\right\}$ (the powers $z^{-q J}$ are "missing" in (1.1)).

Corollaries. (i) One has

$$
\operatorname{Var}_{A} \mu=\int_{A}|d \mu(z)|=\lim _{r \uparrow 1} \int_{A_{r}}\left|g^{\prime}(z)\right||d z| .
$$

(ii) If $\gamma^{\prime}$ is a closed subarc of an open analytic (or merely rectifiable) arc in $\gamma$, and $g^{\prime}$ has a continuous extension to $D \cup \gamma^{\prime}$, then

$$
\int_{\gamma^{\prime}}|d \mu(z)|=\int_{\gamma^{\prime}}\left|g^{\prime}(z)\right||d z| .
$$

Proof of the lemma. By a theorem of Walsh [6, 8], all continuous functions $f$ on $\bar{D}$ that are holomorphic on $D$ are uniform limits on $\bar{D}$ (hence on $\gamma$ ) of polynomials. Thus, the condition

$$
\int_{\gamma} z^{n} d \mu(z)=0, n=0,1,2, \ldots
$$

implies in particular that

$$
\int_{\gamma} \Phi(z)^{k} d \mu(z)=0, k=0,1,2, \ldots
$$

We now introduce the measure $v=\mu \circ \Psi$ on $\Gamma_{1}=\Gamma$. Then

$$
0=\int_{\Gamma} w^{k} d v(w)=\int_{0}^{2 \pi} e^{i k t} d v\left(e^{i t}\right), k=0,1,2, \ldots:
$$

the measure $v\left(e^{i t}\right)$ has all its Fourier coefficients with non-positive index equal to zero. Thus, by the theorem of $F$. and M. Riesz $[5, c f .1,4]$,

$$
d v(w)=d h(w),|w|=1,
$$


with $h\left(e^{i t}\right)$ absolutely continuous and

$$
h(w)=\sum_{1}^{\infty} a_{n} w^{n} \quad \text { for } \quad|w| \leqslant 1 ;
$$

the derivative $h^{\prime}(w)$ being equal to the Poisson integral of $h^{\prime}\left(e^{i t}\right)$ on $\Delta$, one has

$$
\int_{0}^{2 \pi}\left|h^{\prime}\left(e^{i t}\right)-h^{\prime}\left(r e^{i t}\right)\right| d t \rightarrow 0 \quad \text { as } \quad r \uparrow 1 .
$$

Going back to $D$, we introduce the holomorphic function $g=h \circ \Phi$, so that $d h=d g$ at corresponding points of $\Delta, D$. Now let $A$ be any Borel set on $\gamma$, and $B=\Phi(A)$. Then $A_{r}=\Psi(r B)$, and the boundary behaviour (2.5) of $h^{\prime}$ readily implies that

$$
\begin{aligned}
\mu(A)=v(B) & =\int_{B} h^{\prime}(w) d w=\lim _{r \uparrow 1} \int_{r B} h^{\prime}(w) d w \\
& =\lim _{r \uparrow 1} \int_{A_{r}} g^{\prime}(z) d z
\end{aligned}
$$

which is (2.1).

Again using (2.5), the condition

$$
0=\int_{\gamma} z^{-p_{k}} d \mu(z)=\int_{\Gamma} \Psi(w)^{-p_{k}} h^{\prime}(w) d w, k=1,2, \ldots
$$

shows that

$$
\int_{\Gamma_{r}} \Psi(w)^{-p_{k}} h^{\prime}(w) d w=\int_{\Gamma}=0, \quad 0<r<1 .
$$

Thus

$$
\int_{\gamma_{r}} z^{-p_{k}} d g(z)=0, \quad k=1,2, \ldots
$$

which establishes part (ii) of the lemma.

Comments on the corollaries. Relation (2.3) is a consequence of (2.1)-or can be proved by the same method. Now let $\tilde{\gamma}$ be an open analytic arc in $\gamma$. Then the mapping function $\Phi$ has a 1-1 holomorphic extension which maps a certain open set $\Omega$ containing $\tilde{\gamma}$ onto an open set $\Phi(\Omega)$ containing $\Phi(\tilde{\gamma})$; on $\Phi(\Omega)$, the inverse $\Psi$ is also holomorphic. It follows that for every closed subarc $w=e^{t t}, \alpha \leqslant t \leqslant \beta$ of $\Phi(\tilde{\gamma})$,

$$
\int_{\alpha}^{\beta}\left|\Psi^{\prime}\left(e^{i t}\right)-r \Psi^{\prime}\left(r e^{i t}\right)\right| d t \rightarrow 0 \quad \text { as } \quad r \uparrow 1 .
$$

This relation (which can be proved also if $\tilde{\gamma}$ is only rectifiable-for example, by comparing $\Psi$ with a suitable conformal map $\Psi_{1}$ of $\Delta$ onto a subdomain $D_{1}$ of $D$ bounded by a rectifiable Jordan curve containing $\tilde{\gamma}, c f$. also [4; p. 158]) suffices to establish (2.4):

$$
\left(\int_{\gamma^{\prime}}|d \mu(z)|=\right) \lim _{r \uparrow 1} \int_{\gamma r^{\prime}}\left|g^{\prime}(z)\right||d z|=\int_{\gamma^{\prime}}\left|g^{\prime}(z)\right||d z| .
$$




\section{Conditions on $\gamma$ under which (1.1) spans $C(\gamma)$}

Let $\gamma$ be a Jordan curve around 0 , and $\mu$ a measure on $\gamma$ orthogonal to the powers (1.1). We wish to impose conditions which force $\mu$, or the determining holomorphic function $g$ on $D$, to be zero. Matters will be arranged so that $\gamma$ behaves very badly in the vicinity of a point where $g$ must be regular. This presents no problem at all if $g$. is a polynomial $\sum b_{j} z^{q_{J}}$ (case where finitely many negative powers $z^{-q_{J}}$ are omitted, cf. [2]).

To get beyond this case, we ask that $\gamma$ behave badly where it comes closest to $z=0$. It is simplest to require that $\gamma$ contain only one point closest to the origin, the point $z=1$, say. A simple sufficient condition for regularity of $g$ at $z=1$ is then given by evenness or oddness of $g$ : the point $z=-1$ is inside $D$ ! Oddness of $g$ is achieved by including all the even powers $z^{-2 k}$ in (1.1) (we may omit all the odd powers $z^{-2 k-1}$ ). More generally, the condition that $\left\{p_{k}\right\}$ have positive density (defined as $\lim k / p_{k}$ ) will guarantee that $z=1$ be a regular point of $g$. Indeed, the (increasing) sequence $\left\{q_{j}\right\}$ complementary to $\left\{p_{k}\right\}$ will then have density $d<1$. Thus by a theorem of Fabry and Pólya [3], every arc of the circle of convergence of the power series (2.2) for $g(z)$, of angular measure $>2 \pi d$, will contain a singular point. It follows that this circle must have radius greater than 1 (otherwise, there would be at least two singular points on the unit circle).

We now specify some conditions of "bad behaviour" of $\gamma$ near $z=1$.

Concrete example. Let us start with a cardioid-type curve,

$$
z=\phi_{0}(t)=\left(1+4 \sin \frac{1}{2} t\right) e^{i t}, \quad 0 \leqslant t \leqslant 2 \pi,
$$

and superimpose an "exponential wiggle" near $z=1$ to define $\gamma$ :

$$
z=\phi(t)=\left\{\begin{array}{l}
\phi_{0}(t), 1 / \log \pi \leqslant t \leqslant 2 \pi \\
\phi_{0}(t)+\left(t \sin e^{1 / t}\right) e^{i t}, 0 \leqslant t \leqslant 1 / \log \pi
\end{array}\right.
$$

Suppose now that $g \neq 0$. Then $g^{\prime}$ has a zero of finite multiplicity $m$ (which may be zero!) at $z=1$. Thus on a small neighbourhood $N$ of $z=1$, it satisfies an inequality of the form

$$
\left|g^{\prime}(z)\right| \geqslant c|z-1|^{m}, c>0 .
$$

We choose an analytic subarc $\gamma^{\prime}$ of $\gamma$ in $N$ corresponding to, say $\delta \leqslant t \leqslant \varepsilon$, where $0<\delta<\varepsilon<1 / \log \pi$. Then

$$
\int_{\gamma^{\prime}}\left|g^{\prime}(z)\right||d z| \geqslant \int_{\delta}^{\varepsilon} c|\phi(t)-1|^{m}\left|\phi^{\prime}(t)\right| d t \geqslant c \int_{\delta}^{\varepsilon} t^{m-1} e^{1 / t}\left|\cos e^{1 / t}\right| d t-O(1) .
$$

However, the right-hand side tends to $\infty$ as $\delta \downarrow 0$, contradicting the boundedness of (2.4).

An alternative condition of bad behaviour on $\gamma$ is that there should be a neighbourhood $N$ of $z=1$ in which every subarc of $\gamma$ has infinite length. If $g \neq 0$, the function $g^{\prime}$ would be regular and its modulus would have a positive lower bound on some such subarc. One would then appeal to (2.3) to obtain a contradiction. 


\section{A spanning set (1.1) where $\left\{p_{k}\right\}$ has density zero}

Let $\gamma^{*}$ be any Jordan curve around 0 with the property that (1.1) is a spanning set for $C\left(\gamma^{*}\right)$ whenever the sequence $\left\{p_{k}\right\}$ has positive density. We will show that for such a curve $\gamma^{*}$, there is also a spanning set (1.1) with $\left\{p_{k}\right\}$ of density zero.

Our final sequence $\left\{p_{k}\right\}$ will be of the following type. For integers

$$
0 \leqslant k_{1} \leqslant k_{2} \leqslant \ldots \leqslant k_{r} \leqslant \ldots
$$

which will be specified below, we define

$$
\left.\begin{array}{l}
p_{k}=k, 0<k \leqslant k_{1} ; p_{k}=2 k, k_{1}<k \leqslant k_{2} ; \ldots ; \\
p_{k}=r k, k_{r-1}<k \leqslant k_{r} ; \ldots
\end{array}\right\}
$$

To define the numbers $k_{r}$, it will be convenient to give a name, $S_{r}$ say, to the closed span in $C\left(\gamma^{*}\right)$ of the powers

$$
z^{n} ; n \geqslant 0 \quad \text { and } \quad z^{-p_{k}}, 1 \leqslant k \leqslant k_{r} .
$$

For $k_{1}$, we choose the smallest integer $\geqslant 0$ such that $z^{-1}$ has distance to $S_{1}$ not exceeding 1. For $k_{2}$, we next take the smallest integer $\geqslant k_{1}$ such that $z^{-1}$ and $z^{-2}$ have distance to $S_{2}$ not exceeding 1/2. Assuming that $k_{1}, \ldots, k_{r-1}$ have been determined, we take for $k_{r}$ the smallest integer $\geqslant k_{r-1}$ such that $z^{-1}, z^{-2}, \ldots, z^{-r}$ all have distance to $S_{r}$ not exceeding $1 / r$. That $k_{r}$ exists follows from the fact that $z^{-1}, \ldots, z^{-r}$ belong to the closed span of the powers

$$
z^{n}, n \geqslant 0 ; z^{-p_{k}}, 1 \leqslant k \leqslant k_{r-1} ; z^{-r k}, k_{r-1}<k<\infty .
$$

(Indeed, for this set, the opposites of the negative exponents have density $1 / r$.)

The above construction leads to a set (1.1) whose closed span $S_{\infty}$ is all of $C\left(\gamma^{*}\right)$, while $\left\{p_{k}\right\}$ has density zero. Indeed, the distance between an arbitrary negative power $z^{-q}$ and $S_{\infty}$ (which contains all $S_{r}$ ) is $\leqslant 1 / r$ for all $r \geqslant q$, and hence $z^{-q} \in S_{\infty}$. Also, it is clear that a sequence $\left\{p_{k}\right\}$ of the form (4.1) must have density zero, whether $k_{r}$ tends to $\infty$ or not.

It is easy to see, incidentally, that $k_{r}$ must tend to $\infty$. For suppose not. Then our present set (1.1) would contain only a finite number of negative powers. However, if no power in $(1.1)$ would have exponent $\leqslant-s(s$ integral, $\geqslant 0)$, then $z^{-s-1}$ could not be in the closed span on $\gamma^{*}$, or $z^{-1}$ would be a uniform limit of polynomials on $\gamma^{*}$, and therefore, also on the interior $D^{*}$ !

\section{References}

1. P. L. Duren, Theory of $H^{p}$ spaces (Acad. Press, New York, 1970).

2. J. Korevaar, and P. Pfluger, "Spanning sets of powers on wild Jordan curves ", Nederl. Akad. Wetensch. Proc. Ser. A, 77 (1974), 293-305.

3. G. Pólya, "Untersuchungen über Lücken und Singularitäten von Potenzreihen ", Math. Z. 29 (1929), 549-640.

4. I. I. Priwalow, Randeigenschaften analytischer Funktionen (VEB Deutscher Verlag der Wiss., Berlin, 1956).

5. F. Riesz, and M. Riesz, “ Úber die Randwerte einer analytischen Funktion ”, Quatrième Congrès des Mathématiciens Scandinaves (Stockholm 1916), Uppsala 1920, 27-44.

6. J. L. Walsh, "Über die Entwicklung einer analytischen Funktion nach Polynomen ", Math. Ann., 96(1927), 430-436.

7. _- "Uber die Entwicklung einer Funktion einer komplexen Veränderlichen nach Polynomen", Math. Ann., 96 (1927), 437-450. 
8. - Interpolation and approximation by rational functions in the complex domain (Amer. Math. Soc. Colloq. Publ., Vol. 20, New York, 1935).

9. J. Wermer, "Nonrectifiable simple closed curve ", Advanced problems and solutions, no. 4687, Amer. Math. Monthly, 64 (1957), 372.

University of California, San Diego. University of Michigan.

University of Amsterdam. 